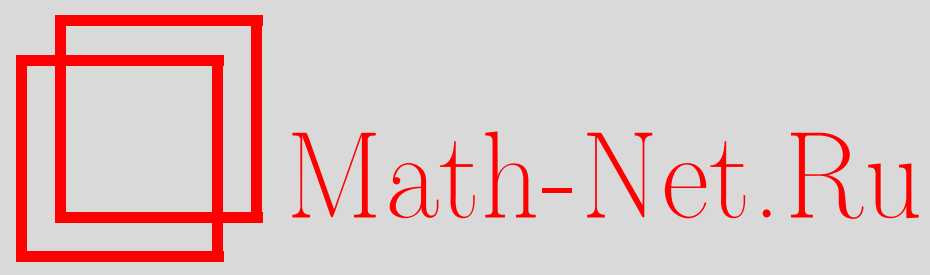

Ю. С. Колесов, Проблема аттракторов нелинейных волновых уравнений в плоских областях, Матем. заметки, 2000, том 68, выпуск 2, 217-229

DOI: https://doi.org/10.4213/mzm940

Использование Общероссийского математического портала Math-Net.Ru подразумевает, что вы прочитали и согласны с пользовательским соглашением http://www . mathnet.ru/rus/agreement

Параметры загрузки:

IP: 34.239 .49 .27

26 апреля 2023 г., $17: 48: 05$

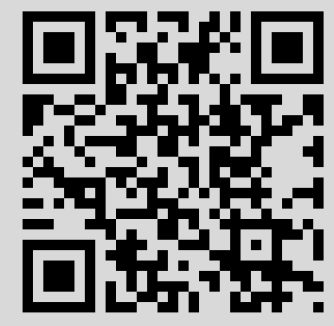




\title{
ПРОБЛЕМА АТТРАКТОРОВ \\ НЕЛИНЕЙНЫХ ВОЛНОВЫХ УРАВНЕНИЙ \\ В ПЛОСКИХ ОБЛАСТЯХ
}

ю. С. Колесов

\begin{abstract}
Рассматривается типовой пример квазилинейного волнового уравнения в единичном квадрате при нулевых граничных условиях. При помощи метода квазинормальных форм показьвается, что из его нулевого состояния равновесия бифуцирует большое число дихотомичных цик лов и торов. Высказьвается гипотеза о возможной структуре аттрактора.

Библиография: 14 названий.
\end{abstract}

Ставится задача о бифуркации автоколебаний типового квазилинейного волнового уравнения в единичном квадрате при нулевых граничных условиях. Использование метода квазинормальных форм приводит к заключению, что из его нулевого состояния равновесия бифуцирует большое число дихотомичньх циклов и торов, причем размерность неустойчивого многообразия каждого из них бесконечна. В связи с этим возникает проблема о его аттракторах. Формулируется гипотеза об их возможной природе.

Метод квазинормальных форм обязан своим созданием проблеме о роли малого миграционного фактора в динамике изменения плотности популящии, обитающей в однородном ареале, пищевая база в котором регулярно восстанавливается до определенного уровня. В математическом плане задача сводится к построению пространственно неоднородных колебаний в однородных средах с малой диффузией. Первые теоремы о бифуркации пространственно неоднородных циклов в системах типа реакция-диффузия получены в [1], [2]. В последующем эти результаты были распространены на случай многочастотных колебаний в [3] (см. также [4], где дано более развернутое обоснование относяшихся сюда утверждений). Отметим также статью [5], в которой в максимально доступной форме предложенньй в [1], [2] подход к вопросу о бифуркации циклов проиллюстрирован на примере одной частной задачи, обладающей такими упрощающими свойствами, что это позволило получить тривиальное обоснование соответствующего предложения.

Примерно параллельно, но вначале менее интенсивно, развивалась техника, позволяюшая применить метод квазинормальных форм к решению бифуркационных проблем для уравнений волнового типа. $\mathrm{K}$ первому из исследованных вопросов относится задача о бифуркации циклов в одной системе уравнений волнового типа при нелинейных граничных условиях [6]. В идейном и техническом отношении много более сложна

Работа выполнена при финансовой поддержке Российского фонда фундаментальных исследований, грант № 97-01-00549. 
задача о распространении метода квазинормальных форм на сингулярно возмущенные уравнения волнового типа. Однако к настоящему времени и здесь имеется определенный прогресс [7], [8].

Ниже затронут новьй вопрос. Именно, при помощи метода квазинормальньх форм предпринимается попытка объяснения, что в случае волновых уравнений их динамические свойства существенно меняются при переходе от одномерной области к плоской.

1. Постановка задачи и базовая лемма. Типовым объектом в исследуемом вопросе мы считаем краевую задачу

$$
u_{t t}-\varepsilon u_{t}+u^{2} u_{t}+\delta^{2} u=\Delta u,\left.\quad u\right|_{\Gamma}=0,
$$

где $\varepsilon$ - малый положительньй параметр, постоянная $\delta>0, \Delta$ - оператор Лапласа, $\Gamma$ - граница единичного квадрата $0 \leqslant x, y \leqslant 1$. Тем самьм в целях простоты и наглядности ограничиваем свое рассмотрение относительно конкретной краевой задачей для классического волнового уравнения с часто используемой в приложениях нелинейностью Ван-дер-полевского типа. В конце статьи, правда, поясним, что на самом деле наш основной результат достаточно универсален.

Если в (1) отбросить нелинейность, решения получающейся линейной краевой задачи растут по экспоненциальному закону с показателем экспоненты порядка $\varepsilon$. Нелинейность гасит этот рост, что приводит к возникновению так назьваемых установившихся колебаний, в качестве которых в случае отрезка служит большое число циклов [9]. Наша цель - показать, что при переходе к плоской области бифурцируюший из состояния равновесия аттрактор не связан с циклами или торами, а значит, можно говорить об открытии нового феномена в теории автоколебаний волновых уравнений.

Фазовым пространством (пространством начальных условий) краевой задачи (1) в дальнейшем считаем произведение соболевских пространств $\stackrel{\circ}{W}_{2}^{2} \times W_{2}^{1}$, где нолик сверху в первом из них означает подчиненность функций нулевьм граничным условиям. Это важное понятие позволяет практически без изменений распространять на уравнения с частными производными основные положения качественной теории обькновенных дифференциальных уравнений.

Для решения поставленной бифуркационной проблемы прибегнем к методу квазинормальных форм. В его основе лежит формализм процедуры построения нормальной формы Пуанкаре-Дюлака, а точнее, его подходящая модификация, учитьвающая специфику краевой задачи (1). Подчеркнем сразу следующее важное обстоятельство: если нелинейность кубическая, то структура младших слагаемых нормальной формы существенно зависит от наличия или отсутствия резонансов третьего порядка, отличных от тождественных.

Итак, предварительно нам следует разобраться с резонансами в линейной краевой задаче

$$
u_{t t}+\delta^{2} u=\Delta u,\left.\quad u\right|_{\Gamma}=0,
$$

колебания в которой происходят с частотами

$$
\omega_{k n}=\pi \sqrt{\delta^{2}+k^{2}+n^{2}}, \quad k, n=1,2, \ldots
$$

Отметим, что $\omega_{k n}^{2}-$ собственные числа оператора $\delta^{2} I-\Delta$, которьм соответствуют нормированные в $L_{2}$ единицей собственные функции

$$
u_{k n}=2 \sin k \pi x \sin n \pi y .
$$


Напомним также, что резонанс третьего порядка называется тождественным, если $\omega_{k n}=\omega_{k n}+\omega_{p s}-\omega_{p s}$. Как прояснится в следующем пункте, нас должны заботить только такие нетождественные резонансы третьего порядка

$$
\omega_{k n}=\omega_{k_{1} n_{1}} \pm \omega_{k_{2} n_{2}} \pm \omega_{k_{3} n_{3}}
$$

что одновременно являются ненулевыми величины

$$
\int_{0}^{1} \int_{0}^{1} u_{k n} u_{k_{1} n_{1}} u_{k_{2} n_{2}} u_{k_{3} n_{3}} d x d y
$$

Последнее означает, что каждое из равенств типа (2) должно дополняться какими-то равенствами вида

$$
k=k_{1} \pm k_{2} \pm k_{3}, \quad n=n_{1} \pm n_{2} \pm n_{3} .
$$

ЛЕммА. При ограничениях (3) не реализуется ни один из нетохдественных резонансов (2).

ДокАЗАТЕЛЬСТво. Начнем с технического замечания. Удобно в $(2)$ вместо $\omega_{n k}$ писать $\omega$, а вместо $\omega_{k_{1} n_{1}}, \omega_{k_{2} n_{2}}, \omega_{k_{3} n_{3}}$ использовать обозначения $\omega_{1}, \omega_{2}, \omega_{3}$ соответственHO.

Естественно, что в первую очередь целесообразно разобраться с наиболее сложным из возможных вариантов. В дальнейшем прояснится, что таковым следует считать случай, когда мы имеем дело с нетождественным резонансом

$$
\omega=\omega_{1}+\omega_{2}-\omega_{3}
$$

дополненным равенствами

$$
k=k_{1}+k_{2}-k_{3}, \quad n=n_{1}+n_{2}-n_{3} .
$$

Введем в рассмотрение четыре вектора в $\mathbb{R}^{3}$

$$
a=(\delta, k, n), \quad a_{j}=\left(\delta, k_{j}, n_{j}\right), \quad j=1,2,3 .
$$

В терминах этих векторов равенство (4) означает, что

$$
\|a\|+\left\|a_{3}\right\|=\left\|a_{1}\right\|+\left\|a_{2}\right\|
$$

где $\|*\|$ - евклидова длина вектора. Из (5) и (6) также следует, что

$$
a+a_{3}=a_{1}+a_{2}
$$

Равенства (7) и (8) позволяют получить соотношение

$$
\begin{gathered}
\alpha \frac{a}{\|a\|}+(1-\alpha) \frac{a_{3}}{\left\|a_{3}\right\|}=\beta \frac{a_{1}}{\left\|a_{1}\right\|}+(1-\beta) \frac{a_{2}}{\left\|a_{2}\right\|}, \\
\alpha=\frac{\|a\|}{\|a\|+\left\|a_{3}\right\|}, \quad \beta=\frac{\left\|a_{1}\right\|}{\left\|a_{1}\right\|+\left\|a_{2}\right\|} .
\end{gathered}
$$


Подведем промежуточньй итог: из (9) и (10) вытекает, что пересекаются принадлежашие единичному шару отрезки, задаваемые соответственно левой и правой частями формулы (9), в которых, правда, в этом случае нужно считать независимыми параметры $\alpha$ и $\beta$. На интуитивном уровне ясно, что данная ситуация маловероятна. Установим строго, что это действительно невозможно.

Предварительно покажем, что не могут совпадать векторы $a_{1}$ и $a_{2}$. Действительно, если $a_{1}=a_{2}$, то равенство (9) преобразуется к виду

$$
\frac{a_{1}}{\left\|a_{1}\right\|}=\alpha \frac{a}{\|a\|}+(1-\alpha) \frac{a_{3}}{\left\|a_{3}\right\|} .
$$

Однако представление (11) для вектора $a_{1}$ возможно в единственном случае, когда

$$
\frac{a}{\|a\|}=\frac{a_{1}}{\left\|a_{1}\right\|}, \quad \frac{a_{3}}{\left\|a_{3}\right\|}=\frac{a_{1}}{\left\|a_{1}\right\|} .
$$

В соответствии с (6) отсюда следует, что $\omega=\omega_{1}, \omega_{3}=\omega_{1}$, а значит, имеем дело с тождественным резонансом, т. е. вступаем в противоречие с исходной посылкой.

Допустим, что векторы

$$
\frac{a}{\|a\|}, \frac{a_{1}}{\left\|a_{1}\right\|}, \frac{a_{2}}{\left\|a_{2}\right\|}, \frac{a_{3}}{\left\|a_{3}\right\|}
$$

принадлежат некоторой двумерной плоскости, проходящей через начало координат. Отсюда следует, что ранг матришы, вектор-столбцами которой служат данные векторы, равен двум. Этот ранг не меняется при умножении вектор-столбцов на положительные числа. Поэтому равен нулю определитель каждой из матриц

$$
\left(\begin{array}{ccc}
1 & 1 & 1 \\
k_{1} & k_{2} & k_{3} \\
n_{1} & n_{2} & n_{3}
\end{array}\right), \quad\left(\begin{array}{ccc}
1 & 1 & 1 \\
k & k_{1} & k_{2} \\
n & n_{1} & n_{2}
\end{array}\right) .
$$

Предположим, что первая из матриц (12) аннулируется на векторе, у которого равна нулю третья координата. Отсюда вьводим, что $a_{1}=a_{2}$, а невозможность данного равенства уже установлена. Еще один вариант: пусть та же матрища аннулируется на векторе, у которого, скажем, равна нулю первая координата. Данное допущение в качестве следствия влечет равенство $\omega_{2}=\omega_{3}$, а это противоречит предположению, что рассматривается нетождественный резонанс.

Меняя в случае необходимости нумерацию векторов (6), можно добиться выполнения неравенств

$$
k_{1}<k_{3}<k_{2}
$$

При таком способе действия на самом деле можно гарантировать справедливость нестрогих неравенств. Однако, из излагаемого ниже станет ясно, что невозможно ни одно из равенств.

Выше нами показано, что все координаты ненулевого вектора, на котором аннулируется первая матрища (12), заведомо ненулевые. При этом в соответствии с неравенствами (13) координаты этого вектора имеют вид $\alpha_{1}, \alpha_{2},-\alpha_{1}-\alpha_{2}$, где $\alpha_{1}, \alpha_{2}>0$, а значит, выполняется соотношение

$$
a_{3}=\frac{\alpha_{1}}{\alpha_{1}+\alpha_{2}} a_{1}+\frac{\alpha_{2}}{\alpha_{1}+\alpha_{2}} a_{2} .
$$


Прояснилась причина, по которой невозможны равенства в (13).

Обратимся ко второй матрице (12). Так как согласно (13) $k_{1}<k<k_{2}$, то она аннулируется только на векторе, координаты которого имеют вид $-\beta_{1}-\beta_{2}, \beta_{1}, \beta_{2}$, где $\beta_{1}, \beta_{2}>0$, а поэтому имеет место соотношение

$$
a=\frac{\beta_{1}}{\beta_{1}+\beta_{2}} a_{1}+\frac{\beta_{2}}{\beta_{1}+\beta_{2}} a_{2} .
$$

Из линейной независимости векторов $a_{1}$ и $a_{2}$, равенства (8) и полученных нами формул (14) и (15) вытекает, что

$$
a_{3}=\gamma a_{1}+(1-\gamma) a_{2}, \quad a=(1-\gamma) a_{1}+\gamma a_{2},
$$

где $0<\gamma<1$. Из (16) следует, что

$$
\left\|a_{3}\right\| \leqslant \gamma\left\|a_{1}\right\|+(1-\gamma)\left\|a_{2}\right\|, \quad\|a\| \leqslant(1-\gamma)\left\|a_{1}\right\|+\gamma\left\|a_{2}\right\| .
$$

Складьвая неравенства (17) и вспоминая о равенстве (7), заключаем, что все четыре вектора (6) совпадают, т. е. снова выходит противоречие.

Через начало координат и точки $a_{1}, a_{2}$ проведем двумерную плоскость. Над этой плоскостью нависает двузвенная ломаная, составленная из векторов $a_{3}$ и $a_{1}+a_{2}-a_{3}$ (последний вектор считаем выходяшим из точки $a_{1}+a_{2}$ ). Путем движения положим ее на введенную двумерную плоскость. Данное построение приводит нас к вьпуклому четырехугольнику, две примыкаюшие стороны которого имеют длины $\left\|a_{1}+a_{2}-a_{3}\right\|$ и $\left\|a_{3}\right\|$, а длины оставшихся сторон соответственно равны $\left\|a_{1}\right\|$ и $\left\|a_{2}\right\|$. В силу равенства (7) построенный четырехугольник с необходимостью является параллелограммом. Отсюда следует, что мы снова имеем дело с тождественным резонансом, а значит, при ограничениях (4) и (5) справедливо утверждение леммы.

Остальные варианты несопоставимо проще. Например, пусть

$$
\begin{gathered}
\omega=\omega_{1}+\omega_{2}+\omega_{3}, \\
k=k_{1}+k_{2}+k_{3}, \quad n=n_{1}+n_{2}+n_{3} .
\end{gathered}
$$

Возводя левую и правую части в (18) в квадрат и учитьвая равенства (19), приходим к некоторому соотношению, которое заведомо не может быть верным. Лемма доказана.

2. Основной результат и его обоснование. Используем асимптотический метод интегрирования Крылова-Боголюбова для построения главной части формальной нормальной формы краевой задачи (1). Реализация этого подхода связана с представлением решений в виде асимптотического ряда

$$
u=\varepsilon^{1 / 2} u_{0}+\varepsilon^{3 / 2} u_{1}+\cdots
$$

где

$$
u_{0}=\sum_{k, n}\left[v_{k n}(\tau) \exp \left(i \omega_{k n} t\right)+\text { к.c. }\right] u_{k n}(x, y) .
$$

Здесь $\tau=\varepsilon t-$ медленное время, а к. с. - обьчное сокрашение для комплексно сопряженной величины. В $(20)$ гладкая по совокупности переменных функция $u_{1}=u_{1}(\tau, t, x, y)$ квазипериодична по $t$ с частотным набором $\omega_{k n}$. Подставим $(20)$ в $(1)$ и приравняем 
коэффишиенты при степенях $\varepsilon^{3 / 2}$. В итоге для отыскания $u_{1}$ получим неоднородное линейное уравнение

$$
\frac{\partial^{2} u_{1}}{\partial t^{2}}+\left(\delta^{2} I-\Delta\right) u_{1}=\frac{\partial}{\partial t}\left(u_{0}-2 \frac{\partial u_{0}}{\partial \tau}-\frac{u_{0}^{3}}{3}\right)
$$

дополненное, естественно, нулевыми граничньми условиями. Отметим, что в $(21) \tau$ рассматривается как параметр. Уравнение (21) имеет квазипериодическое по $t$ решение в том и только том случае, если, во-первых, вьполняется соотношение

$$
\begin{aligned}
2 \frac{d v_{k n}}{d \tau}= & v_{k n}-\frac{9}{4} v_{k n}\left|v_{k n}\right|^{2}-2 v_{k n} \sum_{\substack{p \neq k \\
s \neq n}}\left|v_{p s}\right|^{2} \\
& -3 v_{k n} \sum_{p \neq k}\left|v_{p n}\right|^{2}-3 v_{k n} \sum_{s \neq n}\left|v_{k s}\right|^{2}, \quad k, n=1,2, \ldots,
\end{aligned}
$$

а во-вторых, с ростом $k$ и $n$ последовательность $\left|v_{k n}\right|$ убьвает достаточно быстро.

Систему уравнений $(22)$, дополненную аналогичной системой для $\bar{v}_{k n}$, назовем $к в a-$ зинормальной формой краевой задачи (1).

Итак, благодаря лемме квазинормальная форма краевой задачи (1) устроена относительно просто. Чтобы прояснить вопрос об ее аттракторах, удобно, полагая $\rho_{k n}=\left|v_{k n}\right|^{2}$, перейти от нее к так назьваемой системе амплитудных уравнений

$$
\frac{d \rho_{k n}}{d \tau}=\left(1-\frac{9}{4} \rho_{k n}-2 \sum_{\substack{p \neq k \\ s \neq n}} \rho_{k s}-3 \sum_{p \neq k} \rho_{p n}-3 \sum_{s \neq n} \rho_{k s}\right) \rho_{k n} .
$$

Будем интерпретировать квазинормальную форму краевой задачи (1) как абстрактное уравнение в $l_{2}^{1}$. В этом случае систему амплитудных уравнений следует рассматривать в таком пространстве последовательностей, в котором сходится двойной ряд с общим членом $\omega_{k n}^{2}\left|\rho_{k n}\right|$. Впрочем, по построению нас должны интересовать аттракторы системы (23), принадлежашие конусу $K_{+}$векторов с неотрицательными координатами.

Примем следуюшее определение [9]. Назовем состояние равновесия системы (23) $m$-мерным, где целое число $m \geqslant 1$, если положительны только какие-то его $m$ координат (из принадлежности $K_{+}$следует, что остальные координаты нулевые).

Достаточно очевидно, что система $(23)$ в $K_{+}$имеет счетное число указанных состояний равновесия. Остановимся на способе выявления их свойств устойчивости.

Пусть для определенности $1 \leqslant k, n \leqslant N_{0}$, где $N_{0} \geqslant 2$. Считая, что в (23) $\rho_{k n}=0$, если $k \geqslant N_{0}+1$ или $n \geqslant N_{0}+1$, приходим к обыкновенному уравнению в $\mathbb{R}^{N_{0}^{2}}$

$$
\frac{d \rho}{d \tau}=\left(e_{0}-A \rho\right) \rho
$$

где $e_{0}$ - вектор, у которого каждая координата равна единице, матрица $A$ с положительными элементами при подходящей нумерации координат вектора $\rho$ является симметричной, умножение векторов в правой части покоординатное. Для примера разберем вопрос об устойчивости внутреннего состояния равновесия уравнения (24), все координаты которого положительны. 
Чтобы дальнейшее изложение было яснее, вьпишем в координатах систему (24) при $N_{0}=2$. Пусть

$$
\rho_{1}=\rho_{11}, \quad \rho_{2}=\rho_{12}, \quad \rho_{3}=\rho_{21}, \quad \rho_{4}=\rho_{22} .
$$

Тогда система (24) имеет вид:

$$
\begin{aligned}
& \dot{\rho}_{1}=\left(1-\frac{9}{4} \rho_{1}-3 \rho_{2}-3 \rho_{3}-2 \rho_{4}\right) \rho_{1}, \\
& \dot{\rho}_{2}=\left(1-3 \rho_{1}-\frac{9}{4} \rho_{2}-2 \rho_{3}-3 \rho_{4}\right) \rho_{2}, \\
& \dot{\rho}_{3}=\left(1-3 \rho_{1}-2 \rho_{2}-\frac{9}{4} \rho_{3}-3 \rho_{4}\right) \rho_{3}, \\
& \dot{\rho}_{4}=\left(1-2 \rho_{1}-3 \rho_{2}-3 \rho_{3}-\frac{9}{4} \rho_{4}\right) \rho_{4},
\end{aligned}
$$

где точка означает дифференцирование по $\tau$.

Заметим, что координаты внутреннего состояния равновесия совпадают (см. в качестве наглядного примера систему (25)), а для их общего значения $\rho_{0}$ справедлива формула:

$$
\rho_{0}=\frac{1}{2 N_{0}^{2}+2 N_{0}-7 / 4} .
$$

Данное обстоятельство упрошает выявление свойств устойчивости состояния равновесия уравнения (24), принадлежащего внутренности первого октанта. Действительно, после нормировки времени уравнение в вариациях принимает простой вид:

$$
\dot{h}=-A h .
$$

Из (27) следует, что нам нужно разобраться со знаками чисел $\mu$, определяемых из системы уравнений

$$
\begin{gathered}
-\frac{1}{4} h_{k n}-2 l(h)-\sum_{p \neq k} h_{p n}-\sum_{s \neq n} h_{k s}=\mu h_{k n}, \\
l(h)=\sum_{k, n=1}^{N_{0}} h_{k n} .
\end{gathered}
$$

Предположим, что $l(h) \neq 0$. Выполняя в (28) суммирование по $k$ и $n$, убеждаемся, что

$$
\mu=-\frac{1}{\rho_{0}} .
$$

Перейдем к варианту, когда

$$
l(h)=0 .
$$

При условии (31) равенство (28) запишем в виде

$$
-\sum_{p=1}^{N_{0}} h_{p n}-\sum_{s=1}^{N_{0}} h_{k s}=\left(\mu-\frac{7}{4}\right) h_{k n} .
$$


Предположим, что не равна нулю по крайней мере одна из сумм, фигурирующих в левой части (32). Пусть таковой будет первая из них. Вьполняя тогда в (32) суммирование по $k$ и вспоминая о формуле (29) и равенстве (31), приходим к вьводу, что

$$
\mu=\frac{7}{4}-N_{0}
$$

Остается рассмотреть вариант, когда при некоторых $k$ и $n$ равна нулю каждая из сумм, стоящая в левой части $(32)$, но при этом $h_{k n} \neq 0$. Заключаем, что

$$
\mu=\frac{7}{4}
$$

Из (30), (33) и (34) следует дихотомичность рассматриваемого состояния равновесия уравнения (24). Отметим, что при этом оно может иметь устойчивые состояния равновесия, лежашие на гранях первого октанта. Например, система (25) имеет в первом октанте экспоненциально устойчивые состояния равновесия

$$
\left(\frac{4}{17} 0,0, \frac{4}{17}\right), \quad\left(0, \frac{4}{17}, \frac{4}{17}, 0\right) .
$$

Поэтому полное представление о свойствах устойчивости $m$-мерных состояний равновесия системы (23) можно получить, работая именно с ней, а не с ее укороченным вариантом (24) или (25).

Предположим, что речь идет о свойствах устойчивости внутреннего состояния равновесия уравнения (24), рассматриваемого теперь в рамках системы (23). В этом случае $\rho_{k n}=0$ при $k \geqslant N_{0}+1$ или $n \geqslant N_{0}+1$. Поэтому при указанных $k$ и $n$ линеаризация соответствующего уравнения системы (23) приводит нас к уравнению

$$
\frac{d h_{k n}}{d \tau}=\left(1-2 m_{1} \rho_{0}-3 m_{2} \rho_{0}-3 m_{3} \rho_{0}\right) h_{k n}
$$

где неотрицательные числа $m_{1}, m_{2}, m_{3}$ в сумме не превосходят $N_{0}^{2}$. Отметим, что коэффициент перед $h_{k n}$ в правой части уравнения (35) в нуль обратиться не может. Действительно, в предположении противного согласно (26) должно выполняться равенство

$$
\frac{7}{4}=2 N_{0}^{2}+2 N_{0}-2 m_{1}-3 m_{2}-3 m_{3}
$$

невозможность которого ясна. Заметим еше, что коэффишиент перед $h_{k n}$ в правой части уравнения (35) может иметь любой знак.

Аналогичным образом выявляются свойства устойчивости состояний равновесия (24), лежаших на каких-то гранях первого октанта.

Фиксируем какое-то $m$-мерное состояние равновесия системы (23), у которого положительны координаты $\rho_{k_{1} n_{1}}, \ldots, \rho_{k_{m} n_{m}}$. В системе $(22)$ ему соответствует состояние равновесия, у которого ненулевыми являются только координаты

$$
v_{k_{1} n_{1}}=\sqrt{\rho_{k_{1} n_{1}}}, \ldots, \quad v_{k_{m} n_{m}}=\sqrt{\rho_{k_{m} n_{m}}} .
$$


Отсюда и из (19), (20) следует, что краевая задача (1) имеет приближенньй инвариантный тор

$$
\begin{gathered}
u_{\text {пр }}(\varphi, x, y, \varepsilon)=\varepsilon^{1 / 2} \sum_{s=1}^{m}\left[v_{k_{s} n_{s}} \exp \left(i \varphi_{k_{s} n_{s}}\right)+\text { к.c. }\right] u_{k_{s} n_{s}}(x, y), \\
\frac{d \varphi}{d t}=\omega
\end{gathered}
$$

где $\varphi$ и $\omega-m$-мерные векторы с компонентами $\varphi_{k_{s} n_{s}}$ и $\omega_{k_{s}} n_{s}$ соответственно.

Для систем обыкновенных дифференпиальных уравнений в [10] разработана схема, позволяющая достаточно просто доказывать существование дихотомичных инвариантных торов в ситуациях, подобных той, с которой мы сталкиваемся при анализе краевой задачи (1). Используем развитьй в [10] подход для доказательства следующего предложения.

ТЕОРемА. Найдется такое $\varepsilon_{0}>0$, что при $0<\varepsilon<\varepsilon_{0}$ краевая задача (1) имеет дихотомичный тор

$$
\begin{gathered}
u(\varphi, x, y, \varepsilon)=u_{\text {пр }}(\varphi, x, y, \varepsilon)+\varepsilon^{3 / 2} u_{\text {ост }}(\varphi, x, y, \varepsilon), \\
\frac{d \varphi}{d t}=\omega+\varepsilon^{2} \omega_{\text {ост }}(\varphi, \varepsilon),
\end{gathered}
$$

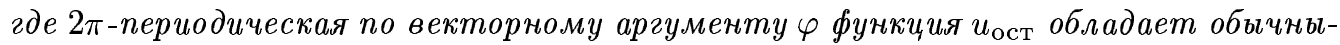
ми для решений волновых уравнений свойствами гладкости, а $2 \pi$-периодическая по $\varphi$ вектор-функиия $\omega_{\text {ост }}$ и ее произвольно фиксированное число производных по $\varphi$ равномерно по є ограничены.

ДокАЗАТЕЛЬСтво. Обозначим через $B$ арифметический квадратньй корень из оператора $\delta^{2} I-\Delta$ при нулевых граничных условиях. Затем стандартным образом перейдем от краевой задачи (1) к эквивалентной системе

$$
\dot{w}=A_{0} w+\varepsilon A_{1} w+F(w)
$$

где $w$-вектор-функция с компонентами $w_{1}=B u, w_{2}=u_{t}$, точка означает дифференцирование по $t$,

$$
A_{0}=\left(\begin{array}{cc}
0 & B \\
-B & 0
\end{array}\right), \quad A_{1}=\left(\begin{array}{cc}
0 & 0 \\
0 & I
\end{array}\right), \quad F(w)=\left(\begin{array}{c}
0 \\
-w_{2}\left(B^{-1} w_{1}\right)^{2}
\end{array}\right) .
$$

Линеаризуя уравнение (39) на приближенном торе (37), (38), получаем

$$
\dot{h}=A_{0} h+\varepsilon A_{1} h+\varepsilon A_{2}(t) h,
$$

где, очевидно, $A_{2}(t)$ - ограниченный в $W_{2}^{1} \times W_{2}^{1}$ оператор, квазипериодически зависящий от $t$, с частотным базисом $\omega_{k_{s}} n_{s}$. Вопрос об устойчивости решений подобных уравнений подробно рассмотрен в [11]. Однако нас будут интересовать тонкости, которые там опущены. 
Воспользуемся изложенньг в [4] алгоритмом исследования устойчивости абстрактных дифференциальных уравнений. Для этого введем в рассмотрение бесконечную матрицу-строку

$$
V_{0}(t)=\left[\ldots, u_{k n}\left(\begin{array}{l}
1 \\
i
\end{array}\right) \exp \left(i \omega_{k n} t\right), u_{k n}\left(\begin{array}{c}
1 \\
-i
\end{array}\right) \exp \left(-i \omega_{k n} t\right), \ldots\right] .
$$

Затем составим выражение

$$
\left(V_{0}(t)+\varepsilon V_{1}(t)\right) \exp (\varepsilon D t)
$$

Отметим, что здесь $V_{1}(t)$ - бесконечная матрица-строка, структурно подобная $V_{0}(t)$, но пока с неизвестными элементами. Нам также пока неизвестны элементы бесконечной в обе стороны матрищы $D$. Для их отыскания подставим выражение (41) в уравнение (40). Приравнивая коэффициенты при $\varepsilon$, убеждаемся, что имеет место соотношение

$$
\dot{V}_{1}=A_{0} V_{1}+A_{1} V_{0}+A_{2} V_{0}-V_{0} D
$$

Потребуем, чтобы элементы матришы $V_{1}$ были квазипериодичны с частотным базисом $\omega_{k n}$. Так как $A_{1}(t)$ - операторньй тригонометрический многочлен, то указанное ограничение позволяет построить матрицу-строку $V_{1}(t)$ при подходящем выборе элементов матрицы $D$ (последнее означает, что за их счет удовлетворяются обычные условия ортогональности).

Первое важное замечание: сопоставляя данный алгоритм со способом вьвода квазинормальной краевой задачи (1), заключаем, что с той же матрицей $D$ мы имеем дело при линеаризации системы $(22)$, дополненной системой для $\bar{v}_{k n}$ таким образом, что переменные $v_{k n}$ и $\bar{v}_{k n}$ чередуются, а линеаризация проводится на состоянии равновесия указанной системы, когда ненулевыми являются только числа (36).

Выполняя в уравнении (40) замену

$$
h=\left(V_{0}+\varepsilon V_{1}\right) p, \quad p \in l_{2}^{1},
$$

приходим к эквивалентному уравнению в $l_{2}^{1}$

$$
\dot{p}=\varepsilon D p+\varepsilon^{2} \Gamma(t, \varepsilon) p,
$$

где норма оператора $\Gamma$ равномерно по $t$ и $\varepsilon$ ограничена и квазипериодична по $t$ с тем же частотным базисом, что и $V_{1}$.

Второе важное замечание: прямая выкладка показьвает, что матрица $D$ аннулируется на векторах

$$
\left(\ldots, 0, i v_{k_{s} n_{s}}-i v_{k_{s} n_{s}}, 0, \ldots\right), \quad s=1, \ldots, m .
$$

Напомним, что (см. (35)) матрица $D$ диагональна, причем двумерные блоки, количество которых равно $m$, связаны с векторами (42). Отсюда следует, что для укороченного уравнения

$$
\dot{p}=\varepsilon D p
$$

имеет место трихотомия решений [4]. Именно, пространство $l_{2}^{1}$ распадается в прямую сумму корневых подпространств $E_{0}, E_{+}, E_{-}$оператора $D$, отвечающих соответственно его нулевым, отрицательным и положительным собственньм значениям. Подчеркнем 
еще раз, что размерность подпространства $E_{0}$ равна $m$, а каждое из подпространств $E_{+}$ и $E_{-}$бесконечномерно.

Третье важное замечание: в силу нашей базисной леммы связанные с индексами $k$ и $n$ элементы матрицы $V_{1}$ содержат в качестве множителей функции

$$
\exp \left(i \omega_{k n} t\right), \quad \exp \left(-i \omega_{k n} t\right)
$$

если

$$
(k, n) \neq\left(k_{s}, n_{s}\right), \quad s=1, \ldots, m .
$$

Данное обстоятельство позволяет нам сначала ввести матрицу-строку $W_{0}(\varphi)$, где $\varphi$ находится из $(38)$, отличающуюся от $V_{0}(t)$ тем, что опущены экспоненты (43) в тех ее элементах, для которых справедливы неравенства (44). После этого из аналогичных соображений составим матрицу-строку $W_{1}(\varphi)$, используя $V_{1}(t)$. Если теперь в уравнении (40) вьполнить замену

$$
h=\left(W_{0}+\varepsilon W_{1}\right) p
$$

то получим уравнение в $l_{2}^{1}$

$$
\dot{p}=J p+\varepsilon D p+\varepsilon^{2} \Gamma(\varphi, \varepsilon) p,
$$

где $J$ - диагональная матрица, на главной диагонали которой чередуются числа $i \omega_{k n}$ и $-i \omega_{k n}$, исключая те наборы $(k, n)$, для которых неравенства (44) преврашаются в равенства (в этом случае соответствуюшие диагональные элементы равны нулю). Поэтому введенные выше подпространства $E_{0}, E_{+}, E_{-}$для оператора $J+\varepsilon D$ выполняют ту же роль, что и для оператора $D$.

В соответствии с (20) учтем в правой части формулы (37) слагаемые порядка $\varepsilon^{3 / 2}$. Связано это с тем, что для доказательства теоремы нам потребуется, чтобы после подстановки приближенного тора $w_{\text {пр }}(\varphi, x, y, \varepsilon)$ в уравнение $(39)$ невязка вьходила порядка $\varepsilon^{5 / 2}$. На этом более точном торе линеаризацию уравнения (39) запишем в виде

$$
\dot{h}=A_{0} h+\varepsilon A_{1} h+\varepsilon A_{2}(\varphi) h+\varepsilon^{2} A_{3}(\varphi, \varepsilon) h,
$$

где $A_{2}$ - тот же оператор, что и в правой части уравнения (49), а $2 \pi$-периодический по $\varphi$ оператор $A_{3}$ и его любое фиксированное число производных по $\varphi$ равномерно по $\varepsilon$ ограничены в $W_{2}^{1} \times W_{2}^{1}$. В дальнейшем конкретный вид этого оператора роли не играет, а значит, допустима его подходящая вариация.

Четвертое (и последнее) важное замечание: в соответствии с изложенным в [4] оператор $A_{3}$ можно так изменить (с сохранением перечисленных чуть вьше свойств гладкости по $\varphi$ ), что при помоши замены (45) уравнение (47) преобразуется к виду (ср. с (46))

$$
\dot{p}=J p+\varepsilon D p .
$$

Из этого замечания вытекает, что для уравнения (47) имеет место трихотомия решений, причем она осушествляется при помощи $2 \pi$-периодических по $\varphi$ проекторов $P_{0}(\varphi, \varepsilon)$ и $P_{ \pm}(\varphi, \varepsilon)$, нормы которых (а также их произвольно фиксированное число производных по $\varphi$ ) равномерно по $\varepsilon$ ограничены. 
Приступим к завершающей стадии доказательства теоремы. В окрестности приближенного тора уравнения (39) обычным образом введем переменные $\varphi$ и $h$ при помощи замены

$$
w=w_{n p}(\varphi, x, y, \varepsilon)+\varepsilon h, \quad h=P h,
$$

где $P=P_{+}(\varphi, \varepsilon)+P_{-}(\varphi, \varepsilon)$. В результате оно преобразуется в систему [3], [4]

$$
\begin{aligned}
\dot{h} & =A(\varphi, \varepsilon) h+\varepsilon^{3 / 2} \Phi(\varphi, h, \varepsilon), \\
\dot{\varphi} & =\omega+\varepsilon^{2} \psi(\varphi, h, \varepsilon),
\end{aligned}
$$

где оператор $\Phi$ и вектор-функция $\psi$ гладко зависят от $\varphi$ и $h$. Отметим, что в правой части уравнения (49) $A$ - действуюший в области значений $P$ линейньй оператор, являющийся сужением линейного оператора, фигурирующего в правой части уравнения (47). При этом предполагаем, что методом “добавить и отнять" оператор $A_{3}$ изменен так, что справедлив факт, составивший содержание четвертого замечания. Отсюда следует, что существование гладкого инвариантного тора у системы (49), (50) может быть установлено при помощи метода последовательных приближений. Правда, разработанная в [10] схема проходит без дополнительных рассуждений, если решения (как в случае обыкновенных уравнений) обладают некоторьми свойствами компактности. Данное затруднение преодолевается следующим образом.

Будем рассматривать уравнение (39) в пространстве $\stackrel{\circ}{W}_{2}^{1+\nu} \times \stackrel{\circ}{W}_{2}^{1+\nu}$, где положительная постоянная $\nu$ произвольно мала. Ясно, что и при таком выборе фазового пространства уравнения (39) справедливо все ранее сказанное. Поэтому имеется требуемая компактность решений - пространство $\stackrel{\circ}{W}_{2}^{1+\nu} \times \stackrel{\circ}{W}_{2}^{1+\nu}$ компактно вложено в $W_{2}^{1} \times W_{2}^{1}$.

Неустойчивость построенного тора устанавливается, например, по предложенному в [3], [4] способу. Теорема доказана.

Сделаем два дополнительных замечания. Во-первых, отметим ключевое значение того факта, что при помоши замены (45) подходящее уравнение вида (47) преобразуется в уравнение (48). Во-вторых, следует подчеркнуть, что дихотомичность инвариантного тора в рамках нелинейного уравнения (39) может быть установлена путем доказательства существования у него устойчивых и неустойчивых многообразий (например, проходит изложенный в [12] способ их конструирования).

3. Заключение. Начнем с описания одного из возможных обобщений. В прямоугольнике $0 \leqslant x \leqslant a, 0 \leqslant y \leqslant b$ рассмотрим краевую задачу

$$
u_{t t}-\varepsilon \mu u_{t}+u=f\left(u, u_{t}\right)+\varepsilon \Delta u,\left.\quad u\right|_{\Gamma}=0 .
$$

Здесь $f$ - гладкая функция, имеющая в нуле высший порядок малости, такая, что отрицательна действительная часть первой ляпуновской величины обькновенного уравнения, в которое трансформируется (51) при $\varepsilon=0$. Если допустить, что положительный параметр $\mu$ относительно мал, к краевой задаче (51) применима не только процедура квазинормализации, но и последуюшая процедура вторичной квазинормализации. Предположим, что при этом $a^{2} / b^{2}$ есть иррациональное число. Тогда при проведении вторичной квазинормализации избегаем почти всех нетождественных резонансов третьего порядка, что позволяет получить (после подходящей нормировки) систему, по своим динамическим свойствам во многом подобную системе (23).

Наконец, коснемся важного вопроса о структуре аттрактора краевой задачи (1). 
ГИПотЕЗА. Существует слохсно устроенное функииональное множество, лежащее вне фазового пространства $\stackrel{\circ}{W}_{2}^{2} \times W_{2}^{1}$ краевой задачи (1), которое притягивает почти все ее решения.

Если говорить проше, то в краевой задаче (1) стохастическое поведение решений сочетается с потерей ими гладкости.

В заключение отметим, что вопрос об аттракторах уравнений волнового типа давно привлекал внимание многих исследователей. Однако пока полученные результаты со многих точек зрения носят “конечномерньй” характер [13], что отражается даже в обычно накладываемых ограничениях [14], исключающих из рассмотрения краевые задачи вида (1).

\section{СПИСОК ЦИТИРОВАННОЙ ЛИТЕРАТУРЫ}

[1] Колесов Ю. С. Задача паразит-хозяин // Динамика биологических популяций. Горький: Изд-во Горьковского ун-та, 1984. С. 16-29.

[2] Колесов Ю.С. Об одной бифуркационной теореме в теории автоколебаний распределенных систем // Дифференц. уравнения. 1985. Т. 21. №10. С. 1709-1713.

[3] Колесов Ю. С. Бифуркация инвариантных торов параболических систем с малой диффузией // Матем. сб. 1993. Т. 184. № 3. С. 121-136.

[4] Мищенко Е. Ф., Колесов Ю. С., Колесов А. Ю., Розов Н. Х. Периодические движения и бифуркационные процессы в сингулярно возмушенных системах. М.: Физматлит, 1995.

[5] Васильева А. Б., Кащенко С.А., Колесов Ю. С., Розов Н. Х. Бифуркация автоколебаний нелинейных параболических уравнений с малой диффузией // Матем. сб. 1986. T. 130. № 4. С. 488-499.

[6] Биркган С.Е. Экспоненциальная дихотомия и устойчивость решений дифференциально-разностных уравнений нейтрального типа с почти периодическими коэффициентами. Дисс. ... к. ф.-м. н. Воронеж: Воронежский гос. ун-т, 1985.

[7] Колесов Ю.С. Асимптотика и устойчивость нелинейных параметрических колебаний сингулярно возмушенного телеграффного управления // Матем. сб. 1995. Т. 186. №10. С. 57-72.

[8] Колесов Ю.С. Параметрические колебания сингулярно возмущенного телеграфного управления с маятниковой нелинейностью // Матем. сб. 1998. Т. 189. № 3. С. 69-82.

[9] Колесов Ю.С. Свойства устойчивости циклов и торов простейшего нерезонансного уравнения волнового типа // Матем. заметки. 1997. Т. 62. № 5. С. 744-750.

[10] Самойленко А. М. Элементы математической теории многочастотных колебаний. Инвариантные торы. М.: Наука, 1987.

[11] Фомин В. Н. Математическая теория параметрического резонанса в линейных распределенных системах. Л.: Изд-во ЛГУ, 1972.

[12] Хартман $\Phi$. Обыкновенные дифференциальные уравнения. М.: Мир, 1970.

[13] Бабин А. В., Вишик М. И. Аттракторы параболических и гиперболических уравнений. Характер их компактности и притяжения к ним // Вестн. МГУ. Сер. 1. Матем., мех. 1988. № 3. C. 71-73.

[14] Горицкий А. Ю. Асимптотика неограниченных при $t \rightarrow \infty$ решений гиперболического уравнения // Вестн. МГУ. Сер. 1. Матем., мех. 1990. № 2. С. 56-58. 